\title{
Notes on relevant, irrelevant, marginal and extremal double trace perturbations
}

\author{
Massimo Porrati and Cedric C.Y. Yu \\ Center for Cosmology and Particle Physics, \\ Department of Physics, New York University, \\ 4 Washington Place, New York, NY 10003, U.S.A. \\ E-mail: mp9@nyu.edu, cyy272@nyu.edu
}

ABSTRACT: Double trace deformations, that is products of two local operators, define perturbations of conformal field theories that can be studied exactly in the large- $N$ limit. Even when the double trace deformation is irrelevant in the infrared, it is believed to flow to an ultraviolet fixed point. In this note we define the Källen-Lehmann representation of the two-point function of a local operator $O$ in a theory perturbed by the square of such operator. We use such representation to discover potential pathologies at intermediate points in the flow that may prevent to reach the UV fixed point. We apply the method to an "extremal" deformation that naively would flow to a UV fixed point where the operator $O$ would saturate the unitarity bound $\Delta=\frac{d}{2}-1$. We find that the UV fixed point is not conformal and that the deformed two-point function propagates unphysical modes. We interpret the result as showing that the flow to the UV fixed point does not exist. This resolves a potential puzzle in the holographic interpretation of the deformation.

KEYwords: AdS-CFT Correspondence, Conformal Field Theory, Renormalization Group

ArXiv ePrint: 1609.00353 


\section{Contents}

1 Introduction 1

2 Multi trace deformations 3

3 Two-point functions of double trace perturbations and their KällenLehmann representation 5

3.1 Case 1: $1>\nu>0 \quad 6$

$\begin{array}{lll}3.1 .1 & \lambda<0 & 7\end{array}$

$\begin{array}{lll}3.1 .2 \lambda>0 & 7\end{array}$

3.2 Case 2: $0>\nu>-1 \quad 8$

$\begin{array}{lll}3.2 .1 & \lambda>0 & 8\end{array}$

$\begin{array}{lll}3.2 .2 \lambda<0 & 8\end{array}$

3.3 Case $3: \nu>1 \quad 9$

3.4 Case $4: \nu=0 \quad 9$

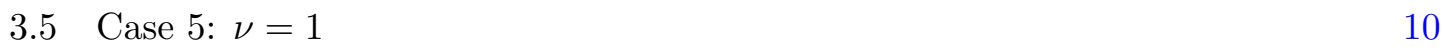

$\begin{array}{lll}4 & \text { Summary } & 12\end{array}$

$\begin{array}{ll}\text { A Alternative quantization in AdS/CFT } & 13\end{array}$

\section{Introduction}

Certain conformal field theories that come in families defined by an integer $N$ simplify in the large- $N$ limit. Notable examples are theories that can be described by weakly coupled gravities via the holographic duality and $O(N)$ vector models. The latter are deformations of $N$ massless free field theories. The deformation is called by abuse of term "double trace." It is defined as $\lambda N O^{2}$, with $O=C \sum_{a=1}^{N} \phi^{a} \phi^{a}$ (for bosons) or $O=C \sum_{a=1}^{N} \bar{\psi}^{a} \psi^{a}$ (for fermions). Choosing $C=\mathcal{O}(1 / N)$ the correct large- $N$ scaling for the coupling $\lambda$ of the double-trace perturbation is $\lambda=\mathcal{O}\left(N^{0}\right)$.

The deformed $O(N)$ models are particularly interesting in three spacetime dimensions. The deformation of the bosonic model is relevant and flows to a nontrivial infrared fixed point $[1,2]$. The deformation of the fermionic model is irrelevant, so non-renormalizable by power counting. Nevertheless, a UV fixed point is believed to exist in the large- $N$ limit [36] and even at finite $N .{ }^{1}$ Both models can be solved exactly by using a method that easily generalizes to the case of adjoint theories with holographic semiclassical gravity duals. In the context of holographic duality, the correct treatment of multi-trace perturbations was explained in [8] and further simplified in [9]. The analysis of [9] was further extended and

\footnotetext{
${ }^{1}$ See [7] for a recent review of evidence in favor of the existence of a UV fixed point.
} 
generalized beyond AdS/CFT holography in [10]. We will review the results of $[9,10]-$ for completeness and to fix notations and normalizations - in section 2.

The rediscovery of multi-trace perturbations in the context of AdS/CFT duality makes clear that they can be studied exactly in an appropriately defined large- $N$ limit, ${ }^{2}$ even when the CFT is not free and the operator $O$ has arbitrary conformal dimension $\Delta$. A feature of the exact solution of the deformed model is that, at the fixed points, the conformal dimension of a deformation which is the product of primary fields $O_{1}, \ldots O_{m}$ is the sum of the individual dimensions $\Delta=\Delta_{1}+\ldots+\Delta_{m}$, but the dimensions of the individual operators at the two fixed points (UV and IR) are in general different.

The possibility of solving the deformed model raises several interesting questions, which we shall try to answer in this paper. One such question arises already in the case of a doubletrace deformation $O^{2}$ when $2 \Delta>d$ in $d$ spacetime dimensions. In this case the deformation is irrelevant in the IR, that is non renormalizable by power counting. Nevertheless the exact solution of the deformed model at large " $N$ " has a UV fixed point. A difficult question is whether this UV fixed point exists at finite $N$. A simpler one is whether the fixed point can be connected to the IR by a physical renormalization group (RG) flow. By "physical" we mean the following: the deformed theory has both an IR and a UV fixed point; therefore, it defines a field theory valid at all energies. The theory is thus a UV complete one rather than merely an effective field theory, valid only up to a maximum energy scale. To qualify as a "physical" this theory must be free of tachyons and ghosts. Of course an "unphysical" theory, plagued by ghosts or tachyons, can still have physical UV or IR fixed points. We will see several examples of such behavior in section 3. Such theory may also describe an interesting statistical mechanics system, but not a relativistic, local field theory. We call an RG flow "physical" when it is generated by a deformation that produces a physical relativistic field theory. Ref. [8] shows that the RG flow due to the double trace perturbation $O^{2}$ connects a fixed point where the scaling dimension of $O$ is $\Delta_{+}=d / 2+\nu$ to one where the dimension is $\Delta_{-}=d / 2-\nu$. In the range $0<\nu<1$ both dimensions satisfy the unitarity bound $\Delta \geq d / 2-1$. Outside the range, $\Delta_{-}$violates the unitarity bound, so the RG flow should be pathological. This turns out to be the case: the pathology shows up in a particular UV completion of the theory because of the presence of unphysical poles in the two-point function of the operator $O$ [11].

We will recover this pathology in section 3 , where we will refine the analysis of refs. $[11,12]$ by giving a complete Källen-Lehmann representation of the two-point correlator $\langle O(x) O(0)\rangle$. Section 3 will also study the case $\nu=1$, which is perhaps the most intriguing of all double-trace perturbations for CFTs that are dual to semiclassical gravity theories. Their bulk dual contains a scalar, $\Phi$, with squared mass $(m L)^{2}=-d^{2} / 2+1$ ( $L=$ AdS radius). The standard quantization of such bulk theory associates to the bulk scalar $\Phi$ an operator $O$ of dimension $d / 2+1$. The RG flow, if it existed, would end in a theory where $O$ had dimension $d / 2-1$, which therefore would saturate the unitarity bound. In a unitary CFT any operator saturating the unitarity bound must be a free field [13]. ${ }^{3}$

\footnotetext{
${ }^{2}$ Here $N$ denotes the order of magnitude of the number of degrees of freedom in the unperturbed CFT. In even dimensions it is given, at least parametrically, by the coefficient of a conformal anomaly.

${ }^{3}$ See also [14], and [15] for a recent review.
} 
This free field should come from a different identification of sources and VEVs in a theory with the same near-boundary behavior of the scalar field and the same bulk action [16]. On the other hand, a standard scalar action does not carry the singleton representation corresponding to a free scalar [17]. ${ }^{4}$ So, an obvious question to ask is whether the flow induced by the "extremal" double trace perturbation $O^{2}$, with $O$ of dimension $d / 2+1$, is physical all the way up to the UV and really terminates in a free-field fixed point. We will be able to answer this question (in the negative) at the end of section 3. Final remarks on operator mixing and connections to other papers on double-trace perturbations — in section 4 - and an appendix summarizing the AdS/CFT holographic description of multi-trace perturbations conclude the paper.

\section{Multi trace deformations}

As a warm-up example of multi-trace deformations let us consider the interacting $O(N)$ vector model. In three dimensions it was conjectured to be the holographic dual of $A d S_{4}$ high spin theories in [19]. Its action is

$$
I_{\mathrm{CFT}}=\int d^{d} x \sum_{a=1}^{N}\left(\frac{1}{2}\left(\partial \phi^{a}\right)^{2}+\frac{\lambda}{2 N}\left(\phi^{a} \phi^{a}\right)^{2}\right), \quad \lambda>0 .
$$

The deformation is relevant in dimension $d<4$.

This action can be expressed in terms of the bilinear $\phi^{a} \phi^{a}$ by introducing an auxiliary field $\Sigma$ :

$$
I_{\mathrm{CFT}}=\int d^{d} x \sum_{a=1}^{N}\left(\frac{1}{2}\left(\partial \phi^{a}\right)^{2}+\lambda\left(\phi^{a} \phi^{a}\right) \Sigma-\frac{1}{2} N \lambda \Sigma^{2}\right)
$$

in which the expectation value $\langle\Sigma\rangle=\left\langle\sum_{a=1}^{N} N^{-1} \phi^{a} \phi^{a}\right\rangle$ is formally $\mathcal{O}\left(N^{0}\right)$, i.e. finite in the large- $N$ limit. Integrating out $\Sigma$ one recovers action (2.1); integrating out $\phi^{a}$ and discarding a $\Sigma$-independent constant, one obtains instead a non-local action for $\Sigma$

$$
S[\Sigma]=\frac{N}{2} \log \operatorname{det}\left[-\partial^{2} / 2+\lambda \Sigma\right]-\frac{N \lambda}{2} \int d^{d} x \Sigma^{2} \equiv N s[\Sigma] .
$$

The "intensive" action $s[\Sigma]$ is independent of $N$. The generator of connected correlators of the operator $\sum_{a=1}^{N} \phi^{a} \phi^{a}, W[J] \equiv N w[J]$ is defined by

$$
Z[J]=e^{N w[J]}=\int[d \Sigma] \exp \left[-\frac{N}{2} \log \operatorname{det}\left[-\partial^{2} / 2+\lambda \Sigma-J\right]+\frac{N \lambda}{2} \int d^{d} x \Sigma^{2}\right] .
$$

It is convenient to define an effective action $\gamma_{\lambda}[O]$, independent of $N$ up to terms $\mathcal{O}(1 / N)$, as the Legendre transform of $w[J]$ :

$$
\gamma_{\lambda}[O]=\int d^{d} x O J-w[J], \quad O(x)=\frac{\delta w}{\delta J(x)} .
$$

\footnotetext{
${ }^{4}$ A dipole action for the singleton on a fixed $A d S_{4}$ background was proposed in [18]. The action is quadratic and propagates no degrees of freedom in the bulk.
} 
In the large- $N$ limit the integration over $\Sigma$ in eq. (2.4) reduces to computing a saddle point. Therefore, the effective action is

$$
\gamma_{\lambda}[O]=\int d^{d} x J O+\frac{1}{2} \log \operatorname{det}\left[-\partial^{2} / 2+\lambda \Sigma\right]-\frac{\lambda}{2} \int d^{d} x(\Sigma+J / \lambda)^{2},
$$

computed at the stationary point in $J$ and $\Sigma$. By computing first the stationary point in $J$, it is easy to find that effective action of the deformed $O(N)$ model is

$$
\gamma_{\lambda}[O]=\gamma_{0}[O]+\frac{\lambda}{2} \int d^{d} x O^{2}
$$

So, the effect of the deformation is additive in the effective action. Notice that this result follows simply from the fact that at large $N$ the integral in $\Sigma$ can be evaluated using the saddle point approximation.

As we mentioned before, $W[J]=N w[J]$ generates connected correlators of the operator $\sum_{a=1}^{N} \phi^{a} \phi^{a}$, which are all $\mathcal{O}(N)$. This is another manner of checking that $w[J]$ is independent of $N$ in the large- $N$ limit. Notice that the field $O$ appearing in the free energy is the expectation value of the normalized operator $\sum_{a=1}^{N} N^{-1} \phi^{a} \phi^{a}$, which differs from the operator sourced by $J$ by the normalization factor $N^{-1}$.

We see that the double-trace perturbation is additive in the effective action at leading order in $1 / N$. This simple result generalizes easily to any multi-trace deformation $N U\left(\sum_{a=1}^{N} N^{-1} \phi^{a} \phi^{a}\right)$, when the function $U(x)$ is independent of $N$ [20], and to any theory admitting a large- $N$ limit [10].

In fact, in all theories with an effective action $\mathcal{O}\left(N^{*}\right)$, with $O$ normalized to be $\mathcal{O}(1)$, the perturbation $\int d^{d} x N^{*} U(O)$ shifts the effective action from the unperturbed value $\Gamma=$ $N^{*} \gamma(O)$ to $\Gamma_{U}(O) \equiv N^{*} \gamma(O)+\int d^{d} x N^{*} U(O)$. Here $N^{*}$ is a (large) number counting the effective degrees of freedom of the theory. In even dimensions, this is proportional to the coefficient one of the the conformal anomalies. For $O(N)$ vector models $N^{*}=N$ while for CFTs with fields in the adjoint representation of a rank- $N$ algebra, such as those that possess holographic duals $N^{*}=\mathcal{O}\left(N^{2}\right)$.

To prove additivity of the multi-trace perturbation we begin by writing the Feynman integral representation of the free energy in Lorentzian signature, using the functional Fourier transform of the Dirac delta function. We will denote by $\phi$ the fundamental fields of the CFT and we will use the notation $\langle A, B\rangle \equiv \int d^{d} x A(x) B(x)$ henceforward.

$$
\exp (-i W[J])=\int[d \phi d t d \Omega] \exp \left[+i I[\phi]-i<N^{*}, U(\Omega)>-i N^{*}<J, \Omega>+i<t, \Omega-O[\phi]>\right] .
$$

The composite operator $O[\phi]$ is normalized so that $\langle O[\phi]\rangle=\mathcal{O}(1)$ for $N^{*} \gg 1$. The functional Fourier transform

$$
\int[d J] \exp \left(-i W[J]+i N^{*}<J, O>\right) \equiv \exp \left(i \Gamma_{U}[O]\right),
$$

defines the functional $\Gamma_{U}[O]$ in the perturbed theory in terms of the unperturbed functional $\Gamma[O]$ as

$$
\exp \left(i \Gamma_{U}[O]\right)=\int[d \phi] \exp \left[i I[\phi]-i<N^{*}, U(O)>\right] \delta[O-O[\phi]]=\exp \left(i \Gamma[O]-i<N^{*}, U(O)>\right) .
$$


So far all manipulations have been formal, but exact in $N^{*}$. In the large $N^{*}$ limit, any theory in which the free energy is $W[J]=N^{*} w[J]+\mathcal{O}(1)$, with $w[J]$ independent of $N^{*}$, possesses two additional properties: 1) $\Gamma[O]=N^{*} \gamma[O]+\mathcal{O}(1)$, with $\gamma[O]$ independent of $N^{*}$;2) $\gamma[O]$ is the Legendre transform of $w[J]$. The first property is obvious and the second follows from the saddle-point approximation of the functional integral (2.9). The second property also identifies $\Gamma[O]_{U}$ with the effective action of the perturbed theory and $\Gamma[O]$ with the effective action of the unperturbed theory.

\section{Two-point functions of double trace perturbations and their Källen- Lehmann representation}

Consider an operator $O$ of general conformal dimension $\Delta=d / 2+\nu$. Unitarity requires $\Delta \geq d / 2-1$ (i.e. $\nu \geq-1$ ); when the bound is saturated, $\Delta=d / 2-1, O$ is necessarily free [13] (see also [14, 15]).

Without any deformation, the connected two-point function of $O$ is

$$
\langle O(x) O(0)\rangle=\frac{K}{|x|^{2(d / 2+\nu)}} \equiv K G_{\nu}(|x|) .
$$

The positive pre-factor $K$ is $\mathcal{O}\left(1 / N^{*}\right)$, when the operator $O$ is normalized as in the previous section. In momentum space, defining $\widetilde{O}(k)=\int d^{d} x e^{-i k \cdot x} O(x)$, the two-point function is $[16]$

$$
\langle\widetilde{O}(k) O(0)\rangle=K \widetilde{G}_{\nu}\left(k^{2}\right)=K C\left(\frac{k^{2}}{4}\right)^{\nu}
$$

for non-integer $\nu$. The coefficient $C=\pi^{d / 2} \Gamma(-\nu) / \Gamma(\nu+d / 2)$ is negative for $\nu \in(2 m, 2 m+1)$ (in particular $1>\nu>0$ ) and positive for $0>\nu \geq-1$ and $\nu \in(2 m+1,2 m+2), m \in \mathbb{Z}$.

The cases where $\nu \in \mathbb{Z}^{+}$need to be considered separately due to the appearance of $\ln k^{2}$ terms. In particular, for $\Delta=d / 2+1$ one finds (see appendix)

$$
\widetilde{G}_{\nu=1}\left(k^{2}\right)=K^{-1}\langle\widetilde{O}(k) O(0)\rangle=C^{\prime} k^{2} \ln \left(k^{2} / \mu^{2}\right)
$$

with $C^{\prime}$ positive for $d>2$ and $\mu$ an arbitrary scale that can be changed by adding a contact term proportional to $k^{2}$.

Similarly, for $\Delta=d / 2$,

$$
\widetilde{G}_{\nu=0}\left(k^{2}\right)=-C^{\prime \prime} \ln \left(k^{2} / \mu^{2}\right), C^{\prime \prime}>0 .
$$

The undeformed effective action is then

$$
\gamma_{0}[O]=\frac{1}{2} \int \frac{d^{d} k}{(2 \pi)^{d}} \widetilde{O}(k) \frac{1}{\widetilde{G}_{\nu}\left(k^{2}\right)} \widetilde{O}(-k) .
$$

Now we add a double-trace deformation to the effective action $\gamma[O]$,

$$
U[O]=+\frac{\lambda}{2 \Lambda^{2 \nu}} O^{2}
$$


where $\lambda$ is dimensionless. The cases $\lambda>0$ and $\lambda<0$ will be considered separately. This deformation is IR-relevant for $\Delta<d / 2$, marginal for $\Delta=d / 2$ and irrelevant for $\Delta>d / 2$. It is tempting to identify $\Lambda$ with the cut-off of the theory; the rest of this section will substantiate such identification.

The deformed effective action becomes

$$
\gamma[O]=+\frac{1}{2} \int \frac{d^{d} k}{(2 \pi)^{d}} \widetilde{O}(k)\left(\frac{1}{\widetilde{G}_{\nu}\left(k^{2}\right)}+\frac{\lambda}{\Lambda^{2 \nu}}\right) \widetilde{O}(-k) .
$$

Thus we obtain the deformed two-point function

$$
\widetilde{G}_{\nu, \lambda}\left(k^{2}\right) \equiv \frac{1}{\frac{1}{\widetilde{G}_{\nu}\left(k^{2}\right)}+\frac{\lambda}{\Lambda^{2 \nu}}} .
$$

\subsection{Case 1: $1>\nu>0$}

It was pointed out in [8] that for $1>\nu>0$ such a double-trace deformation leads to an RG flow in which the IR and UV fixed points are CFTs in which the operator $O$ has conformal dimension $\Delta=\Delta_{ \pm}=d / 2 \pm \nu$ respectively. They are the two possible choices of quantization in the AdS/CFT context for a massive scalar in the bulk [16] (see appendix for a review). This flow can be achieved with $\lambda$ positive or negative. Recall that, omitting a positive coefficient, $\widetilde{G}_{\nu}\left(k^{2}\right)=-k^{2 \nu}$. In the IR regime, $k^{2 \nu} \ll \Lambda^{2 \nu} /|\lambda|$,

$$
\begin{aligned}
\widetilde{G}_{\nu, \lambda}\left(k^{2}\right) & =-k^{2 \nu} \frac{1}{1-\frac{\lambda k^{2 \nu}}{\Lambda^{2 \nu}}} \\
& \approx-k^{2 \nu}\left(1+\frac{\lambda k^{2 \nu}}{\Lambda^{2 \nu}}\right) \\
& \approx-k^{2 \nu} \\
& =\widetilde{G}_{\nu, \lambda=0}\left(k^{2}\right)
\end{aligned}
$$

which reduces to the original undeformed CFT of $\Delta=\Delta_{+}$, as expected of an irrelevant deformation.

In the UV, on the other hand, $k^{2 \nu} \gg \Lambda^{2 \nu} /|\lambda|$,

$$
\begin{aligned}
\widetilde{G}_{\nu, \lambda}\left(k^{2}\right) & =\frac{\Lambda^{2 \nu}}{\lambda} \frac{1}{1-\frac{\Lambda^{2 \nu}}{\lambda k^{2 \nu}}} \\
& \approx \frac{\Lambda^{2 \nu}}{\lambda}\left(1+\frac{\Lambda^{2 \nu}}{\lambda k^{2 \nu}}\right) \\
& =\left(\frac{\Lambda^{2 \nu}}{\lambda}\right)^{2} \frac{1}{k^{2 \nu}}+\text { contact terms } \\
& \propto \widetilde{G}_{-\nu, \lambda=0}\left(k^{2}\right) .
\end{aligned}
$$

Upon removing the contact term, this is the two-point function of a CFT with $\Delta=\Delta_{-}$.

Now we come to the heart of our paper. We express the two-point function in KällenLehmann form. 
3.1.1 $\lambda<0$

Consider first $\lambda<0$.

$$
\begin{aligned}
\widetilde{G}_{\Delta, \lambda<0}\left(k^{2}\right) & =-\frac{1}{\frac{1}{k^{2 \nu}}+\frac{|\lambda|}{\Lambda^{2 \nu}}} \\
& =-\frac{\Lambda^{2 \nu}}{|\lambda|}\left(1-\frac{1}{|\lambda|\left(k^{2} / \Lambda^{2}\right)^{\nu}+1}\right) \\
& =+\frac{\Lambda^{2 \nu}}{|\lambda|}\left(\frac{1}{|\lambda|\left(k^{2} / \Lambda^{2}\right)^{\nu}+1}\right)+\text { contact terms. }
\end{aligned}
$$

Let us introduce now the complex function $f(z)=\frac{1}{|\lambda| z^{\nu}+1}$; when its branch cut is placed on the negative real axis it is meromorphic in the range $(\pi \geq \arg z>-\pi)$.

In this case $f(z)$ has no singularity in the first sheet. This implies that there are no tachyonic or otherwise unphysical one-particle states among the states created by applying $O(x)$ to the vacuum. Using Cauchy's formula, one finds

$$
\widetilde{G}_{\nu, \lambda<0}\left(k^{2}\right)=+\frac{\Lambda^{2 \nu}}{\pi} \int_{0}^{\infty} \frac{d m^{2}}{k^{2}+m^{2}} \frac{\left(m^{2} / \Lambda^{2}\right)^{\nu} \sin \pi \nu}{1+\lambda^{2}\left(m^{2} / \Lambda^{2}\right)^{2 \nu}+2|\lambda|\left(m^{2} / \Lambda^{2}\right)^{\nu} \cos \pi \nu} .
$$

So, the spectral density is positive-finite and the spectrum is free of ghosts and tachyons. Thus the deformation with $\lambda<0$ may provide a healthy flow between the IR and UV fixed points. Of course there is a (nonperturbative) fly in the ointment here, since $\lambda<0$ means that the potential $\lambda O^{2}$ is unbounded from below.

\subsection{2 $\lambda>0$}

Consider next the case where $\lambda>0$ :

$$
\widetilde{G}_{\nu, \lambda>0}\left(k^{2}\right)=\frac{\Lambda^{2 \nu}}{\lambda}\left(\frac{1}{\lambda\left(k^{2} / \Lambda^{2}\right)^{\nu}-1}\right),
$$

which has a pole of positive residue at $k^{2} / \Lambda^{2}=\lambda^{-1 / \nu}$ on the positive real axis, signaling a (non-ghost) tachyon mode. The Källen-Lehmann representation shows that the continuum part of the spectral density is positive definite, so the tachyon is the only unphysical feature of the deformed theory:

$$
\begin{aligned}
\widetilde{G}_{\Delta, \lambda>0}\left(k^{2}\right)= & \frac{\Lambda^{2 \nu}}{\lambda} \frac{1}{\nu \lambda^{1 / \nu}\left(k^{2} / \Lambda^{2}-\lambda^{-1 / \nu}\right)} \\
& +\frac{1}{\pi} \int_{0}^{\infty} \frac{d m^{2}}{k^{2}+m^{2}} \frac{m^{2 \nu} \sin \pi \nu}{1+\lambda^{2}\left(m^{2} / \Lambda^{2}\right)^{2 \nu}-2 \lambda\left(m^{2} / \Lambda^{2}\right)^{\nu} \cos \pi \nu} .
\end{aligned}
$$

Two limits are worth mentioning. The first is $\Lambda \rightarrow \infty$. In this case the tachyon moves to infinite mass and the perturbation disappears (since it becomes irrelevant at all energy scales).

The second limit is less trivial. It is the UV limit in which $\Lambda \rightarrow 0$. One interesting question is whether the UV limit may exist as a CFT even if the deformation leading to it 
is unphysical. The answer is yes, because in that limit

$$
\begin{aligned}
\widetilde{G}_{\nu, \lambda>0}\left(k^{2}\right) & \approx \frac{\Lambda^{2 \nu+2}}{\lambda} \frac{1}{\nu \lambda^{1 / \nu} k^{2}}+\frac{1}{\pi} \int_{0}^{\infty} \frac{d m^{2}}{k^{2}+m^{2}} \frac{\Lambda^{4 \nu} m^{2 \nu} \sin \pi \nu}{\lambda^{2} m^{4 \nu}} \\
& \approx \frac{1}{\pi} \int_{0}^{\infty} \frac{d m^{2}}{k^{2}+m^{2}} \frac{\Lambda^{4 \nu} \sin \pi \nu}{\lambda^{2} m^{2 \nu}} .
\end{aligned}
$$

In other words, the tachyonic mode decouples.

\subsection{Case 2: $0>\nu>-1$}

One natural question to ask is: do we get a flow similar to the one above by adding a double-trace deformation for the operator associated to the alternative quantization $d / 2>$ $\Delta>d / 2-1(0>\nu>-1) ?$

In this case

$$
U[O]=+\frac{\lambda}{2 \Lambda^{2 \nu}} O^{2}
$$

is IR-relevant (and UV-irrelevant).

3.2.1 $\lambda>0$

For $\lambda>0$,

$$
\widetilde{G}_{\nu, \lambda>0}\left(k^{2}\right)=\frac{1}{\frac{1}{k^{2 \nu}}+\frac{\lambda}{\Lambda^{2 \nu}}}=-\frac{\Lambda^{2 \nu}}{\lambda} \frac{1}{\lambda\left(k^{2} / \Lambda^{2}\right)^{\nu}+1}+\text { contact terms. }
$$

In Källen-Lehmann form,

$$
\widetilde{G}_{\nu, \lambda>0}\left(k^{2}\right)=+\frac{\Lambda^{2 \nu}}{\pi} \int_{0}^{\infty} \frac{d m^{2}}{k^{2}+m^{2}} \frac{\left(m^{2} / \Lambda^{2}\right)^{\nu}(-\sin \pi \nu)}{1+\lambda^{2}\left(m^{2} / \Lambda^{2}\right)^{2 \nu}+2 \lambda\left(m^{2} / \Lambda^{2}\right)^{\nu} \cos \pi \nu} .
$$

Hence, the spectral density is positive-finite and the spectrum is free of ghosts and tachyons, thus providing a flow between the IR and UV fixed points. One can check that the IR fixed point of this flow is a CFT with an operator with $\Delta=d / 2+|\nu|$, while at the UV fixed point $\Delta=d / 2-|\nu|$. This is expected because the double-trace deformation is UV-irrelevant.

3.2.2 $\lambda<0$

Now for $\lambda<0$,

$$
\begin{aligned}
\widetilde{G}_{\Delta, \lambda<0}\left(k^{2}\right)= & \frac{1}{\frac{1}{k^{2 \nu}}-\frac{|\lambda|}{\Lambda^{2 \nu}}} \\
= & -\frac{\Lambda^{2 \nu}}{|\lambda|} \frac{1}{|\lambda|\left(k^{2} / \Lambda^{2}\right)^{\nu}-1}+\text { contact terms } \\
= & +\frac{\Lambda^{2 \nu}}{|\lambda|} \frac{1}{|\nu||\lambda|^{1 / \nu}\left(k^{2} / \Lambda^{2}-|\lambda|^{-1 / \nu}\right)} \\
& +\frac{1}{\pi} \int_{0}^{\infty} \frac{d m^{2}}{k^{2}+m^{2}} \frac{m^{2 \nu}(-\sin \pi \nu)}{1+\lambda^{2}\left(m^{2} / \Lambda^{2}\right)^{2 \nu}-2|\lambda|\left(m^{2} / \Lambda^{2}\right)^{\nu} \cos \pi \nu} .
\end{aligned}
$$

The two-point function has a pole of positive residue at $k^{2} / \Lambda^{2}=|\lambda|^{-1 / \nu}$ on the positive real axis, signaling a (non-ghost) tachyon mode, while the smooth part of the spectral density is positive definite. 


\subsection{Case 3: $\nu>1$}

The case $\nu>1 \notin \mathbb{Z}^{+}$has been studied in [11] and [12]. Those papers consider a UV completion of the double-trace perturbation obtained by coupling a massive scalar to $O$. In our analysis we do not introduce any such scalar or any other ad hoc UV completion. We use instead a Källen-Lehmann representation, which can be obtained from those used in the previous subsection by replacing $\left(k^{2} / \Lambda^{2}\right)^{\nu}$ with $\pm\left(k^{2} / \Lambda^{2}\right)^{\nu}$, depending on the value of $\nu$.

For $\nu \in(2 m+1,2 m+2), m \in \mathbb{Z}^{+}$with deformation $\frac{\lambda}{2 \Lambda^{2 \nu}} O^{2}$,

$$
\widetilde{G}_{\nu, \lambda}\left(k^{2}\right)=\frac{1}{+\frac{1}{k^{2 \nu}}+\frac{\lambda}{\Lambda^{2 \nu}}}=-\frac{\Lambda^{2 \nu}}{\lambda} \frac{1}{\lambda\left(k^{2} / \Lambda^{2}\right)^{\nu}+1}+\text { contact terms. }
$$

The continuous part of the spectral density is positive-definite for both $\lambda>0$ and $\lambda<0$.

For $\lambda>0$, the simple poles appear at complex values of $k^{2} / \Lambda^{2}$ in conjugate pairs. The corresponding residues also form complex conjugate pairs, signaling tachyonic ghost modes.

For $\lambda<0$, there is exactly one simple pole at real positive $k^{2} / \Lambda^{2}$, with negative residue, i.e. tachyonic ghost.

These results are in agreement with the results of [12] for $2>\nu>1$.

On the other hand, for $\nu \in(2 m, 2 m+1), m \in \mathbb{Z}^{+}$,

$$
\widetilde{G}_{\nu, \lambda}\left(k^{2}\right)=\frac{1}{-\frac{1}{k^{2 \nu}}+\frac{\lambda}{\Lambda^{2 \nu}}}=+\frac{\Lambda^{2 \nu}}{\lambda} \frac{1}{\lambda\left(k^{2} / \Lambda^{2}\right)^{\nu}-1}+\text { contact terms. }
$$

The spectral density is again positive-definite for any $\lambda$.

For $\lambda>0$, there is exactly one simple pole at real positive $k^{2} / \Lambda^{2}$, with positive residue, i.e. non-ghost tachyon.

For $\lambda<0$, the simple poles are again at complex values of $k^{2} / \Lambda^{2}$ and appear in conjugate pairs with, conjugate residues. At least one pair has a negative real part, signaling a tachyonic ghost modes.

Therefore, the deformed theory is not physical, as expected because at the putative UV fixed point the operator $O$ would have dimension $\Delta_{-}=d / 2-\nu$, which is outside the unitary range.

\subsection{Case 4: $\nu=0$}

Next we consider the case $\Delta=d / 2$. The two-point function is $\widetilde{G}_{\nu=0}\left(k^{2}\right)=-\ln \left(k^{2} / \mu^{2}\right)$. The appearance of the scale $\mu$ does not break conformal invariance, since rescaling $k$ amounts only to changing the free energy $w[J]$ by a contact term $\sim J \cdot J$.

Introducing a (marginal) double-trace deformation $U[O]=+\frac{\lambda}{2} O^{2}$, we have

$$
\widetilde{G}_{\nu=0, \lambda}\left(k^{2}\right)=\frac{1}{-\frac{1}{\ln \left(k^{2} / \mu^{2}\right)}+\lambda} .
$$

The renormalization scale $\mu$ can be removed, as in $[8,10]$, by making the coupling constant $\lambda$ run with $\mu$. A convenient renormalization condition on $\lambda$ is to require that it diverges at some fixed scale $\Lambda$. This defines an RG flow of $\lambda$

$$
\lambda \rightarrow \lambda(\mu)=+\frac{1}{\ln \left(\Lambda^{2} / \mu^{2}\right)}
$$


and so

$$
\begin{aligned}
\widetilde{G}_{\nu=0, \lambda}\left(k^{2}\right) & =\frac{1}{-\frac{1}{\ln \left(k^{2} / \mu^{2}\right)}+\frac{1}{\ln \left(\Lambda^{2} / \mu^{2}\right)}} \\
& =\ln \left(\Lambda^{2} / \mu^{2}\right)-\ln ^{2}\left(\Lambda^{2} / \mu^{2}\right) \frac{1}{\ln \left(\Lambda^{2} / k^{2}\right)} .
\end{aligned}
$$

By performing the wave function renormalization $O=Z O_{R}, Z=\ln \left(\Lambda^{2} / \mu^{2}\right)$, we obtain a $\mu$-independent renormalized two-point function

$$
\widetilde{G}_{\nu=0, \lambda}^{(\mathrm{ren})}\left(k^{2}\right)=-\frac{1}{\ln \left(\Lambda^{2} / k^{2}\right)} .
$$

There is a simple pole at $k^{2}=\Lambda^{2}$ with positive residue, i.e. a tachyon mode. This is expected because this theory has a Landau pole for $\lambda>0$ at $\Lambda$ under our renormalization condition. For $\mu>\Lambda$, the coupling constant $\lambda(\mu)$ is negative and asymptotically free, while $\lambda$ is positive and the theory is IR free for $\mu<\Lambda$. All of this is of course in agreement with well known results for the $\lambda \phi^{4}$ theory in four dimensions.

\subsection{Case 5: $\nu=1$}

Now consider the special case, $\Delta=d / 2+1$.

Recall that

$$
\widetilde{G}_{\nu=1}\left(k^{2}\right)=+k^{2} \ln \left(k^{2} / \mu^{2}\right) .
$$

The two-point function of the alternative quantization $\Delta=d / 2-1$ obtained by a naive Legendre transformation (see appendix) is

$$
\widetilde{G}_{\nu=-1}^{(?)}\left(k^{2}\right)=-\frac{1}{k^{2} \ln \left(k^{2} / \mu^{2}\right)} .
$$

Notice that the value of $\mu$ in equation (3.26) does not spoil scale invariance, since it can be changed by adding a contact terms. Instead eq. (3.27) is not scale invariant, since $\mu$ cannot be removed by local counterterms. Moreover, the two-point function of an operator saturating the unitarity bound is

$$
\widetilde{G}_{\nu=-1}\left(k^{2}\right)=\frac{1}{k^{2}} .
$$

So, eq. (3.27) is not the two point function of a $\Delta=d / 2-1$ conformal field. In fact it is altogether unphysical, because it decays faster than $1 / k^{2}$ at large $k^{2}$. The origin of this unphysical feature can be seen by representing the two-point function in Källen-Lehmann form, because such representation makes it manifest that there exists a simple pole at $k^{2}=\mu^{2}$ with negative residue:

$$
\widetilde{G}_{\nu=-1}^{(?)}\left(k^{2}\right)=-\frac{1}{k^{2}-\mu^{2}}+\mathcal{O}\left(\left(k^{2}-\mu^{2}\right)^{0}\right) .
$$

In other words, the spectrum contains a tachyonic ghost mode. Notice that $\mu$ is a physical scale, not an auxiliary one that can be removed by local counterterms. In fact $\mu$ is physical even at the UV fixed point, as pointed out earlier. 
Using Cauchy's formula one obtains

$$
-\frac{1}{k^{2} \ln \left(k^{2} / \mu^{2}\right)}=-\frac{1}{k^{2}-\mu^{2}}+\int_{0}^{\infty} d m^{2} \frac{1}{k^{2}+m^{2}} \frac{1}{\left(m^{2} / \mu^{2}\right)\left(\ln ^{2}\left(m^{2} / \mu^{2}\right)+\pi^{2}\right)}
$$

with

$$
\int_{0}^{\infty} \frac{d m^{2}}{m^{2}\left(\ln ^{2} m^{2}+\pi^{2}\right)}=1
$$

Now add the double-trace deformation $U[O]=\frac{\lambda}{2 \Lambda^{2}} O^{2}$.

For $\lambda>0$,

$$
\begin{aligned}
& \widetilde{G}_{\nu=1, \lambda>0}\left(k^{2}\right) \\
= & \frac{1}{\frac{1}{k^{2} \ln \left(k^{2} / \mu^{2}\right)}+\frac{\lambda}{\Lambda^{2}}} \\
= & -\frac{\Lambda^{2}}{\lambda} \frac{1}{1+\lambda \frac{k^{2}}{\Lambda^{2}} \ln \left(k^{2} / \mu^{2}\right)}+\text { contact terms } \\
= & \text { TWO SIMPLE POLES }+\int_{0}^{\infty} d m^{2} \frac{1}{k^{2}+m^{2}} \frac{m^{2}}{\pi^{2}+\left(1-\left(\frac{\lambda}{\Lambda^{2}}\right) m^{2} \ln \left(m^{2} / \mu^{2}\right)\right)^{2}} .
\end{aligned}
$$

The branch-cut in the complex $k^{2} / \Lambda^{2}$ plane is logarithmic. The spectral density is positivedefinite, but now there are two simple poles.

For $\lambda \mu^{2} / \Lambda^{2}>e$, the poles are at real and positive $k^{2}$. The pole at larger $k^{2}$ has a negative residue and that at the smaller $k^{2}$ a positive residue, with the latter pole approaching 0 as $\lambda \mu^{2} / \Lambda^{2} \rightarrow \infty$.

For $\lambda \mu^{2} / \Lambda^{2}<e$, the poles are complex and are conjugates of each other. The residues have a positive real part and complex conjugate imaginary parts.

For $\lambda<0$,

$$
\begin{aligned}
\widetilde{G}_{\nu=1, \lambda<0}\left(k^{2}\right) & =\frac{1}{\frac{1}{k^{2} \ln \left(k^{2} / \mu^{2}\right)}-\frac{|\lambda|}{\Lambda^{2}}} \\
& =-\frac{\Lambda^{2}}{|\lambda|} \frac{-|\lambda| \frac{k^{2}}{\Lambda^{2}} \ln \left(k^{2} / \mu^{2}\right)}{1-|\lambda| \frac{k^{2}}{\Lambda^{2}} \ln \left(k^{2} / \mu^{2}\right)} \\
& =+\frac{\Lambda^{2}}{|\lambda|} \frac{1}{1-|\lambda| \frac{k^{2}}{\Lambda^{2}} \ln \left(k^{2} / \mu^{2}\right)}+\text { contact terms }
\end{aligned}
$$

which again decays faster than $1 / k^{2}$ at large $k^{2}$ for any $|\lambda| / \Lambda^{2} \neq 0$, so it is again unphysical.

A contour integration gives

$$
\widetilde{G}_{\Delta=d / 2+1, \lambda<0}\left(k^{2}\right)=\text { ONE SIMPLE POLE }+\int_{0}^{\infty} d m^{2} \frac{1}{k^{2}+m^{2}} \frac{m^{2}}{\pi^{2}+\left(1+\left(\frac{|\lambda|}{\Lambda^{2}}\right) m^{2} \ln \left(m^{2} / \mu^{2}\right)\right)^{2}}
$$

in which the spectral density in the second term is positive. The pole is at real and positive $k^{2}$ with negative residue, signaling the propagation of a ghost tachyon mode. 
Therefore, one concludes that the flow to the theory with $\Delta=d / 2-1$ in the UV is unphysical for all values of $\lambda$. Moreover, the very fact that the two-point function in eq. (3.27) is non-unitary shows that the UV limit $\Lambda \rightarrow 0$ is meaningless in this case.

The singleton point is reached the limit $\lambda \rightarrow 0, \mu \exp \left(-1 / \lambda^{2}\right)=$ constant. This limit does decouple all ghost and physical states and leads to a two-point function $\propto 1 / k^{2}$, but it cannot be achieved as an RG trajectory. A different singular limit leading to the singleton is described in [21].

\section{Summary}

After reviewing the method that allows to find the two-point function of certain primary operators $O$ in large- $N$ theories deformed by interactions proportional to $O^{2}$, we studied the RG flow that is determined by the deformation. To get an understanding that goes beyond what is available in the (vast) existing literature on the subject, we used the KällenLehmann representation of the two-point function. This representation allows for a clear and unambiguous detection of unphysical features such as tachyons or ghosts. The presence of such features has a natural interpretation in the case $\nu<-1$, where one of the possible dimensions for the operator $O, \Delta_{-}=d / 2-\nu$, violates the unitarity bound $\Delta \geq d / 2-1$.

We also found that the Källen-Lehmann representation automatically contains extra massive scalars, signaled by simple scalar poles in the two-point function. We can say that the Källen-Lehmann representation "integrates in" massive scalar. One result of our study is that, when the perturbation $O^{2}$ is relevant, the extra massive scalar found using the Källen-Lehmann representation is physical when $\lambda>0$ and tachyonic when $\lambda<0$. The example of the bosonic $O(N)$ vector model in three dimensions shows that this is the expected behavior, because the potential $(\lambda / 2 N)\left(\sum_{a=1}^{N} \phi^{a} \phi^{a}\right)^{2}$ is stable only for $\lambda>0$. On the other hand, the massive scalar used in $[11,12]$ to define a UV completion of doubletrace deformations has the opposite behavior: it is tachyonic for $\lambda>0$ and physical for $\lambda<0$. One possible reason for the disagreement is that the UV completion used in $[11,12]$ can be pathological in the IR. This is manifest in the case of the $O(N)$ vector model, where the scalar potential is unbounded below for either sign of $\lambda$.

An especially interesting case is $\nu=1$, because the conformal dimensions allowed by the alternative quantization of ref. [16] appears to saturate the unitarity bound $\Delta=d / 2-1$. This would be a free field that does not have a dual in a putative semiclassical AdS gravity in $d+1$ dimensions. The flow generated by a double trace perturbation would define a theory where $\Delta=d / 2+1$ flows to the problematic UV fixed point with $\Delta=d / 2-1$. We found that the flow is unphysical, because the two-point function of the operator $O$ always contains unphysical states and the UV fixed point itself is unphysical.

Finally we should remark that our analysis agrees with ref. [22], which studies doubletrace deformations involving two different operators. Here we will restrict our analysis to the most interesting case that one of the two operators, $O_{1}$, saturates the unitarity bound $\left(\Delta_{1}=d / 2-1\right)$ while the other, $O_{2}$, has dimension $\Delta_{2}>\Delta_{1}, \Delta_{2}<d / 2$. Ref. [22] studies a relevant flow from the UV, where $O_{1}$ saturates the unitarity bound, to the IR. It is thus quite different from the situation considered in this paper, which considers an irrelevant 
flow to a putative UV fixed point. Nevertheless, the flow can be studied easily using the methods described in this paper. The deformation studied in [22] is

$$
N \int d^{d} x f O_{1} O_{2}+g_{1} O_{1}^{2}+g_{2} O_{2}^{2}, \quad f, g_{1}, g_{2} \in \mathbb{R} .
$$

One can check that when $g_{1} g_{2}>f^{2}, g_{1}>0, g_{2}>0$ the flow is physical; ${ }^{5}$ on the other hand, whenever $g_{1} \neq 0$ one is simply giving a mass to a free scalar, so the flow is rather trivial: a massive scalar decouples in the IR. So, let us consider the case $g_{1}=0$.

At large $N$ the deformation changes the two point functions $\left\langle\tilde{O}_{i}(k) O_{j}(0)\right\rangle, i, j=1,2$ as

$$
\left\langle\tilde{O}_{i}(k) O_{j}(0)\right\rangle=\left(\begin{array}{cc}
k^{2} & f \\
f & k^{d-2 \Delta_{2}}+g_{2}
\end{array}\right)^{-1}=\frac{1}{k^{d+2-2 \Delta_{2}}+g_{2} k^{2}-f^{2}}\left(\begin{array}{cc}
k^{d-2 \Delta_{2}}+g_{2}-f \\
-f & k^{2}
\end{array}\right) .
$$

In the extreme infrared, $k^{2\left(1+d / 2-\Delta_{2}\right)} \ll f^{2}$

$$
\left\langle\tilde{O}_{i}(k) O_{j}(0)\right\rangle=-\frac{k^{d-2 \Delta_{2}}}{f^{2}}\left(\begin{array}{cc}
1 & -\frac{k^{2}}{f} \\
-\frac{k^{2}}{f} & \frac{k^{4}}{f^{2}}
\end{array}\right)+\text { contact terms. }
$$

The matrix in (4.3) has rank one and is independent of $g_{2}$, meaning that $O_{1}=f \square O_{2}$ in the extreme infrared, where $O_{2}$ has dimension $d-\Delta_{2}$. All this is in perfect agreement with ref. [22]. We conclude by observing that, while some of the UV-complete theories with $g_{1}>0, g_{2}>0$ are physical, those with $g_{1}=0$ are plagued by unphysical states, since the two point function in (4.2) has, among other unpleasantnesses, a tachyonic pole for any value of $g_{2}$. The RG flow generated by the deformation (4.1) with $g_{1}=0$ is therefore unphysical, according to our general definition.

\section{Acknowledgments}

We thank M. Bertolini for useful comments. C.Y.Y. and M.P. are supported in part by NSF grant PHY-1316452.

\section{A Alternative quantization in AdS/CFT}

In this appendix we review the standard $A d S_{d+1} / C F T_{d}$ correspondence and the relationship between the two choices of quantization explained in [16]. Euclidean $A d S_{d+1}$ admits the Poincaré metric

$$
d s^{2}=\frac{1}{z^{2}}\left(d z^{2}+\sum_{i=1}^{d} d x_{i}^{2}\right)
$$

where we set the $A d S_{d+1}$ radius to one. A scalar field $\phi(z, \vec{x})$ of mass $m$ in the bulk has the asymptotic form near the boundary $(z \ll 1)$

$$
\phi(z, \vec{x})=z^{d-\Delta}\left(\phi_{0}(\vec{x})+O\left(z^{2}\right)\right)+z^{\Delta}\left(\frac{A(\vec{x})}{2 \Delta-d}+O\left(z^{2}\right)\right)
$$

in which $\Delta=\Delta_{ \pm}=\frac{d}{2} \pm \sqrt{\frac{d^{2}}{4}+m^{2}} \equiv \frac{d}{2} \pm \nu$ for $\Delta \neq d / 2, \nu \notin \mathbb{Z}$.

\footnotetext{
${ }^{5}$ We thank M. Bertolini for pointing this out to us.
} 
Evaluating the on-shell action and discarding possible contact terms, one finds for $\Delta=\Delta_{+}>d / 2$

$$
\begin{aligned}
S_{A d S_{d+1}}\left[\phi_{0}\right] & =-\frac{1}{2} \int d^{d} x \phi_{0}(x) A(x) \\
& =-\frac{1}{2}(2 \Delta-d) \pi^{-d / 2} \frac{\Gamma(\Delta)}{\Gamma(\Delta-d / 2)} \int d^{d} x \int d^{d} x^{\prime} \frac{\phi_{0}(x) \phi_{0}\left(x^{\prime}\right)}{\left|x-x^{\prime}\right|^{2 \Delta}} .
\end{aligned}
$$

In the case $\Delta=d / 2$,

$$
\phi(z, \vec{x})=z^{d / 2}\left(\ln \left(z / z_{0}\right) \phi_{0}(\vec{x})+A(\vec{x})+O\left(z^{2}\right)\right) .
$$

This asymptotic behavior implies that [23]

$$
S_{A d S_{d+1}}\left[\phi_{0}\right]=-\frac{1}{2} \frac{\Gamma(d / 2)}{2 \pi^{d / 2}} \int d^{d} x \int d^{d} x^{\prime} \phi_{0}(\vec{x}) \frac{1}{\left|\vec{x}-\overrightarrow{x^{\prime}}\right|^{d}} \phi_{0}(\vec{x})
$$

The AdS/CFT correspondence then reads

$$
\exp \left(-N^{*} S_{A d S_{d+1}}\left[\phi_{0}\right]\right)=\left\langle\exp \left(-N^{*} \int \phi_{0} O\right)\right\rangle_{C F T_{d}}=\int[d O] e^{-I_{C F T_{d}}[O]-\int N^{*} \phi_{0} O} .
$$

We have multiplied the exponent by a factor of $N^{*} \gg 1$ such that $\phi_{0}$ and $O$ are both $\mathcal{O}(1)$. For the $\mathrm{O}(\mathrm{N})$ model, $N^{*}=N$.

The corresponding operator $O(x)$ is of conformal dimension $\Delta=\Delta_{+}$. Unitarity requires $\Delta \geq d / 2-1[17]$.

By virtue of eqs. (A.3), (A.5), we identify $-N^{*} S_{A d S_{d+1}}\left[\phi_{0}\right]$ with $W[J]$, the generating functional of connected correlators of the boundary CFT defined in the main body of the paper. The boundary field $\phi_{0}(x)$ is identified with the source $J$ of the boundary conformal operator $O(x)$ as

$$
J=-\phi_{0}
$$

and

$$
O=+\frac{\delta S_{A d S_{d+1}}\left[\phi_{0}\right]}{\delta \phi_{0}}=-A
$$

The two-point function of $O(x)$ can be immediately read off from the action. For $\Delta>d / 2$

$$
N^{*}\langle O(x) O(0)\rangle=(2 \Delta-d) \pi^{-d / 2} \frac{\Gamma(\Delta)}{\Gamma(\Delta-d / 2)} \frac{1}{|x|^{2 \Delta}} \equiv G_{\nu}(|x|)
$$

and for $\Delta=d / 2, N^{*}\langle O(x) O(0)\rangle=\frac{\Gamma(d / 2)}{2 \pi^{d / 2}} \frac{1}{|x|^{2 \Delta}}$.

The two-point function in momentum-space representation in the case $\Delta=d / 2+1$ contains gamma functions in the prefactor that appear divergent, see eq. (3.2). However, 
one notes that

$$
\begin{aligned}
& \left(\frac{d}{d k^{2}}\right)^{2} N^{*}\langle\widetilde{O}(k) O(0)\rangle \\
= & \left(\frac{d}{d k^{2}}\right)^{2}\left[\left((2 \Delta-d) \pi^{-d / 2} \frac{\Gamma(\Delta)}{\Gamma(\Delta-d / 2)}\right) \int d^{d} x e^{-i k \cdot x} \frac{1}{x^{2 \Delta}}\right]_{\Delta=d / 2+1} \\
= & 2 \pi^{-d / 2} \Gamma(d / 2+1) \int d^{d} x e^{-i k \cdot x} \frac{1}{x^{2((d / 2+1)-2)}} \\
= & 2 \pi^{-d / 2} \Gamma(d / 2+1) \int d^{d} x e^{-i k \cdot x} \int_{0}^{\infty} \frac{d t}{t} t^{d / 2-1} e^{-x^{2} t} / \Gamma(d / 2-1) \\
= & 2 \pi^{-d / 2} \Gamma(d / 2+1) \int_{0}^{\infty} \frac{d t}{t} t^{d / 2-1}\left(\frac{\pi}{t}\right)^{d / 2} e^{-\frac{k^{2}}{4 t}} / \Gamma(d / 2-1) \\
s= & \frac{1}{t} \pi^{-d / 2} \Gamma(d / 2+1) \frac{\pi^{d / 2} \int_{0}^{\infty} \frac{d s}{s} s e^{-\frac{k^{2}}{4} s}}{\Gamma(d / 2-1)} \\
= & 2 d(d-2) \frac{1}{k^{2}} .
\end{aligned}
$$

Integrating twice, getting rid of contact terms, and introducing a fictitious scale $\mu$, one arrives at

$$
\widetilde{G}_{\nu=1}\left(k^{2}\right)=N^{*}\langle\widetilde{O}(k) O(0)\rangle=2 d(d-2) k^{2} \ln \left(k^{2} / \mu^{2}\right),
$$

with a positive prefactor for $d>2$.

For $d / 2+1 \geq \Delta>d / 2$, the alternative quantization in which $\Delta=\Delta_{-}$is also allowed by unitarity. To obtain a correspondence to another CFT where the operator $O$ has conformal dimension $\Delta_{-}$, one needs to exchange the roles of $\phi_{0}$ and $A$. Since $\phi_{0}$ and $A$ are conjugate variables, the exchange is done by a Legendre transformation [16].

Define the effective "intensive" action

$$
\gamma[O]=S_{A d S_{d+1}}\left[\phi_{0}\right]-\phi_{0} O
$$

such that

$$
\frac{\delta(\gamma[O])}{\delta O}=-\phi_{0}
$$

Recall that

$$
-\widetilde{A}(k)=\widetilde{O}=+\frac{\delta S_{A d S_{d+1}}\left[\widetilde{\phi_{0}}\right]}{\delta \widetilde{\phi_{0}}}=-\widetilde{G}_{\nu}\left(k^{2}\right) \widetilde{\phi_{0}}(-k),
$$

the effective action of the (undeformed) CFT is

$$
\begin{aligned}
\gamma_{0}[\widetilde{A}] & =+\frac{1}{2} \int \frac{d^{d} k}{(2 \pi)^{d}} \widetilde{A}(k) \frac{1}{\widetilde{G}_{\nu}\left(k^{2}\right)} \widetilde{A}(-k) \\
& =-\frac{1}{2} \int \frac{d^{d} k}{(2 \pi)^{d}} \widetilde{A}(k) \widetilde{G}_{-\nu}\left(k^{2}\right) \widetilde{A}(-k) .
\end{aligned}
$$

Instead of interpreting $\gamma_{0}[\tilde{A}]$ as the effective action for $O$, one can interpret it as the free energy of the CFT operator $O^{\prime}$ of conformal dimension $\Delta_{-}$because the two-point function in the alternative quantization is $\widetilde{G}_{-\nu}\left(k^{2}\right)=-1 / \widetilde{G}_{\nu}\left(k^{2}\right)$. 
Open Access. This article is distributed under the terms of the Creative Commons Attribution License (CC-BY 4.0), which permits any use, distribution and reproduction in any medium, provided the original author(s) and source are credited.

\section{References}

[1] E. Brézin and D.J. Wallace, Critical behavior of a classical Heisenberg ferromagnet with many degrees of freedom, Phys. Rev. B 7 (1973) 1967 [INSPIRE].

[2] K.G. Wilson and J.B. Kogut, The Renormalization group and the $\epsilon$-expansion, Phys. Rept. 12 (1974) 75 [inSPIRE].

[3] D.J. Gross and A. Neveu, Dynamical Symmetry Breaking in Asymptotically Free Field Theories, Phys. Rev. D 10 (1974) 3235 [InSPIRE].

[4] G. Parisi, The Theory of Nonrenormalizable Interactions. 1. The Large-N Expansion, Nucl. Phys. B 100 (1975) 368 [INSPIRE].

[5] B. Rosenstein, B.J. Warr and S.H. Park, The Four Fermi Theory Is Renormalizable in (2+1)-Dimensions, Phys. Rev. Lett. 62 (1989) 1433 [InSPIRE].

[6] G. Gat, A. Kovner, B. Rosenstein and B.J. Warr, Four Fermi Interaction in (2+1)-dimensions Beyond Leading Order in 1/N, Phys. Lett. B 240 (1990) 158 [InSPIRE].

[7] L. Fei, S. Giombi, I.R. Klebanov and G. Tarnopolsky, Yukawa CFTs and Emergent Supersymmetry, arXiv:1607.05316 [INSPIRE].

[8] E. Witten, Multitrace operators, boundary conditions and AdS/CFT correspondence, hep-th/0112258 [INSPIRE].

[9] W. Mueck, An Improved correspondence formula for AdS/CFT with multitrace operators, Phys. Lett. B 531 (2002) 301 [hep-th/0201100] [INSPIRE].

[10] S. Elitzur, A. Giveon, M. Porrati and E. Rabinovici, Multitrace deformations of vector and adjoint theories and their holographic duals, JHEP 02 (2006) 006 [hep-th/0511061] [INSPIRE].

[11] T. Andrade and D. Marolf, AdS/CFT beyond the unitarity bound, JHEP 01 (2012) 049 [arXiv:1105.6337] [INSPIRE].

[12] T. Andrade, T. Faulkner and D. Marolf, Banishing AdS Ghosts with a UV Cutoff, JHEP 05 (2012) 011 [arXiv:1112.3085] [INSPIRE].

[13] D. Buchholz and K. Fredenhagen, Dilations and Interaction, J. Math. Phys. 18 (1977) 1107 [INSPIRE].

[14] S. Weinberg, Minimal fields of canonical dimensionality are free, Phys. Rev. D 86 (2012) 105015 [arXiv: 1210 .3864] [INSPIRE].

[15] D. Simmons-Duffin, TASI Lectures on the Conformal Bootstrap, arXiv:1602.07982 [INSPIRE].

[16] I.R. Klebanov and E. Witten, AdS/CFT correspondence and symmetry breaking, Nucl. Phys. B 556 (1999) 89 [hep-th/9905104] [INSPIRE].

[17] P. Breitenlohner and D.Z. Freedman, Stability in Gauged Extended Supergravity, Annals Phys. 144 (1982) 249 [INSPIRE]. 
[18] M. Flato and C. Fronsdal, The Singleton Dipole, Commun. Math. Phys. 108 (1987) 469 [INSPIRE].

[19] I.R. Klebanov and A.M. Polyakov, AdS dual of the critical $O(N)$ vector model, Phys. Lett. B 550 (2002) 213 [hep-th/0210114] [INSPIRE].

[20] D.J. Amit and E. Rabinovici, Breaking of Scale Invariance in $\phi^{6}$ Theory: Tricriticality and Critical End Points, Nucl. Phys. B 257 (1985) 371 [INSPIRE].

[21] T. Ohl and C.F. Uhlemann, Saturating the unitarity bound in $A d S / C F T_{A d S}$, JHEP 05 (2012) 161 [arXiv:1204.2054] [INSPIRE].

[22] V. Bashmakov, M. Bertolini, L. Di Pietro and H. Raj, Scalar Multiplet Recombination at Large- $N$ and Holography, JHEP 05 (2016) 183 [arXiv: 1603.00387] [INSPIRE].

[23] D.Z. Freedman, S.D. Mathur, A. Matusis and L. Rastelli, Correlation functions in the CFT(d)/AdS(d+1) correspondence, Nucl. Phys. B 546 (1999) 96 [hep-th/9804058] [INSPIRE]. 